\title{
X-ray Emission from Hot DA White Dwarfs; EXOSAT Results, and Implications for Atmospheric Models
}

\author{
Frits Paerels and John Heise \\ Laboratory for Space Research \\ Beneluxlaan 21, 3527 HS Utrecht, the Netherlands
}

\begin{abstract}
We present the observations of the photospheric X-ray spectra of hot DA white dwarfs, obtained with the 500 lines $\mathrm{mm}^{-1}$ Transmission Grating Spectrometer on EXOSAT. These spectra cover the full soft $\mathrm{X}$-ray band, at high wavelength resolution and statistical quality. They allow us to do an accurate measurement of the photospheric parameters, particularly of effective temperature and chemical composition of the atmosphere.

We consider the case of $\mathrm{HZ} 43$ in some detail. Model atmospheric spectra that satisfy all measured absolute optical, UV and X-ray fluxes turn out not to fit the shape of the measured X-ray spectrum. However, from a comparison of model spectra calculated with different model atmospheres codes we infer the existence of a $15 \%$ systematic uncertainty in the model fluxes at the shortest wavelengths $(\lambda<100$ $\AA$ ) in current model calculations. This can explain the fitting problem. Since the systematic uncertainty in the models is larger than the statistical uncertainty in the shape of the measured X-ray spectrum of $\mathrm{HZ} 43$, we cannot at present use this measured shape to derive the effective temperature and gravity. We revert to broad band photometry, using the measured integrated soft X-ray flux and the optical flux, to determine $T_{e}=45,000-54,000 K, R / R_{\odot}=0.0140-0.0165$. From the absence of the He II Ly edge at $227 \AA$ in the measured spectrum, we set a upper limit on the photospheric helium abundance of $\mathrm{He} / \mathrm{H}=$ $1.0 \times 10^{-5}$; this upper limit is independent of the uncertainties in the model calculations mentioned above.
\end{abstract}

\section{X-rays from Hot DA White Dwarfs}

The shape of the photospheric soft X-ray spectrum, and the total soft X-ray flux of hot DA white dwarfs are very sensitive to the photospheric parameters. The total X-ray flux is a steep function of effective temperature, and trace amounts of elements other than hydrogen will produce strong absorption edges in the X-ray/EUV spectrum (at high temperatures, these elements will be highly ionized, and the strong ground state absorption edges and the resonance lines will be in the extreme short-wavelength range). In contrast, the optical and UV spectrum becomes increasingly insensitive to the photospheric parameters with increasing effective temperature.

Soft X-ray observations are therefore obviously of great value to the observational investigation of a number of fundamental questions concerning the physics of hot white dwarfs. Improved estimates of $T_{e}$, stellar radius, and surface gravity from combined X-ray/UV/optical spectroscopy should provide the information to construct luminosity functions and evolutionary sequences. Mass estimates obtained from the measured gravity and radius can be used to experimentally verify theoretical mass-radius relations.

$\mathrm{X}$-ray spectroscopy and photometry can reveal trace amounts of helium and metals in a hot, hydrogen dominated white dwarf atmosphere that are a factor 100 below the present optical spectroscopic detection limit, and so provide us with the means to study the chemical composition of the atmosphere in detail. In principle, the shape of the short-wavelength tail of the stellar spectrum is even sensitive to a possible stratification of elements in the outer envelope of the star. This is 


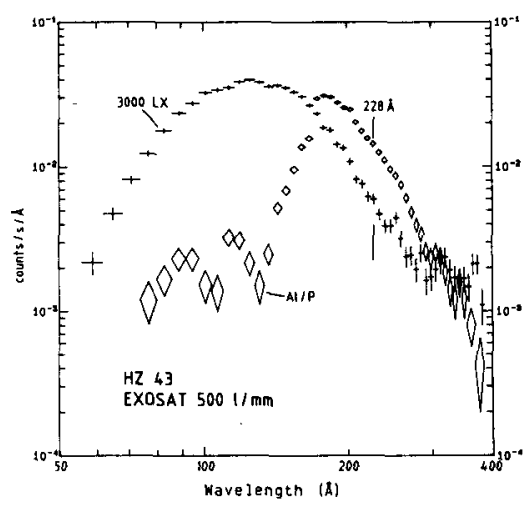

Figure 1. X-ray spectrum of $\mathrm{HZ} 43$, measured with the $500 \mathrm{lmm}^{-1}$ TGS, with the $3000 \mathrm{LX}$ and $\mathrm{Al} / \mathrm{P}$ filters (background subtracted, positive and negative order spectra summed; error bars are $1 \sigma$ photon counting errors). Location of the He II Ly edge has been indicated.

important information, because the atmosphere acts as a thermal blanket to the degenerate core, and hence influences the cooling rate of a white dwarf.

\section{X-ray Observations of Hot White Dwarfs with EXOSAT}

With the launch of the European X-ray Observatory EXOSAT, the Low Energy Imaging Telescopes and Transmission Grating Spectrometers (TGS) of which had a higher sensitivity and a larger dynamic range in the soft X-ray/EUV band than previous experiments (see Taylor [1985], de Korte et al. [1981]), it became possible to measure the X-ray spectrum with high sensitivity between 44 and $400 \AA$, with typical spectral resolution $6 \AA$. Comparison with model atmospheres spectra shows that we are thus able to observe the full $X$-ray spectrum of a hot white dwarf, between the intrinsic cutoff at short wavelengths, to the interstellar absorption cutoff in the EUV band.

We wanted to verify explicitly the conformity of the shape of the X-ray spectrum of hot DA white dwarfs to model atmospheres calculations, and to use the sensitivity of the X-ray spectrum to the stellar parameters to obtain improved estimates of these. We obtained X-ray spectroscopy of three hot DA's, HZ 43, Sirius B, and Feige 24. These are very bright X-ray/EUV sources, and they have been studied extensively in all wavelength bands. This made them the natural 'first choice' for observation with the EXOSAT Transmission Grating Spectrometers.

\section{High Resolution Soft X-ray Spectroscopy of HZ 43}

In the following, we will concentrate on the interpretation of the $\mathrm{HZ} 43$ data. Apart from the fact that HZ 43 has an intrinsic interest as a typical hot DA and a bright X-ray/EUV source, its measured $\mathrm{X}$-ray spectrum has an important implication for current model atmospheres calculations for hot, hydrogen-rich atmospheres. The reader interested in the Sirius B and Feige 24 data is directed to Paerels et al. (1988), and Paerels et al. (1986a), respectively.

HZ 43 was observed with the 500 lines $\mathrm{mm}^{-1}$ TGS on 28 june, 1983, for a total of $18,500 \mathrm{sec}$, with two different beam filters, the 3000LX and $\mathrm{Al} / \mathrm{P}$ filters. The measured spectra are shown in Figure 1 as they appear in the spectrometer (counts $\mathrm{s}^{-1} \AA^{-1}$ vs. wavelength).

The position of the He II Ly edge at $227 \AA$ has been indicated; no edge is detected in the Al/P spectrum (which has the highest statistical quality at the longer wavelengths). This absence can be converted into a sensitive upper limit on the fractional abundance of helium, $\mathrm{He} / \mathrm{H}$. We fitted model atmospheres spectra (which included the He II Ly series and edge) for varying $\mathrm{He} / \mathrm{H}$ to the measured $\mathrm{Al} / \mathrm{P}$ spectrum. $T_{e}$ was fixed at $60,000 \mathrm{~K}, \log g$ at 8.5 ; these values were chosen 

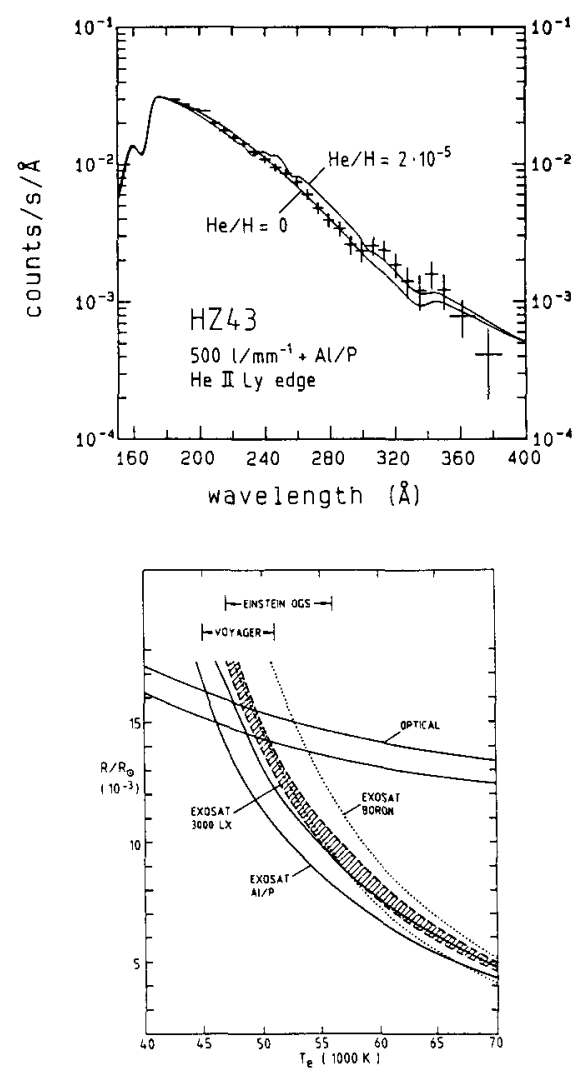

Figure 2. Al/P spectrum of $\mathrm{HZ} 43$ between 170 and $380 \AA$, together with model atmospheres spectra at $T_{e}=$ $60,000 \mathrm{~K}, \log g=8.5, n_{H}=1.4 \times 10^{18} \mathrm{~cm}^{-2}, \mathrm{He} / \mathrm{H}=0$ and $2 \times 10^{-5}$, convolved with the spectrometer response. Best fit is for $\mathrm{He} / \mathrm{H}=0\left(\chi^{2} / 26=1.25\right), 99 \%$ confidence upper limit is at $\mathrm{He} / \mathrm{H}=1.0 \times 10^{-5}$.
Figure 3. 99\% Confidence constraints imposed on $T_{e}$ and $R / R_{\odot}$ of $\mathrm{HZ} \mathbf{4 3}$ by the measured total X-ray flux (in the $3000 \mathrm{LX}$ and $\mathrm{Al} / \mathrm{P}$ spectra, and in photometric observation with the Boron filter), and by the visual magnitude. Intersection of X-ray and 'optical' constraint areas indicates parameter range for which consistent solution to all measured absolute fluxes is found, in terms of a single DA model spectrum. Horizontal bars at the top indicate $99 \%$ confidence estimates of $T_{e}$ from EINSTEIN OGS and Voyager 2 spectra at $R / R_{\odot}=0.015$.

to represent conservative upper limits on these parameters, yielding an equally conservative upper limit on $\mathrm{He} / \mathrm{H}$ (the size of the He II edge decreases with increasing $T_{e}$ and $\log g$ ). The best fit is at $\mathrm{He} / \mathrm{H}=0\left(\chi^{2} / 26=1.25\right)$, the $99 \%$ confidence upper limit is at $\mathrm{He} / \mathrm{H}=1.0 \times 10^{-5}$ (by number), as derived from standard $\chi^{2}$ analysis. Figure 2 shows the $\mathrm{Al} / \mathrm{P}$ spectrum in the region around the edge, together with a model spectrum at $\mathrm{He} / \mathrm{H}=0$, and at $\mathrm{He} / \mathrm{H}=2 \times 10^{-5}$, both convolved with the spectrometer response. This last model was chosen to demonstrate that such a value for $\mathrm{He} / \mathrm{H}$ is already emphatically excluded by the measured shape of the spectrum of $\mathrm{HZ} 43$. The column density of absorbing neutral interstellar gas was determined likewise from the $\mathrm{Al} / \mathrm{P}$ spectrum, and was found to be in the range $n_{H}=6-16 \times 10^{17} \mathrm{~cm}^{-2}$, dependent on effective temperature, with a typical uncertainty of 0.4 in $\log n_{H}(99 \%)$.

Allowing for the ranges in $\mathrm{He} / \mathrm{H}$ and $n_{H}$ given above, and taking values of $\log g$ between 8 and 9 , we determined the constraints imposed on $T_{e}$ and stellar radius $R / R_{\odot}$ by the integrated X-ray flux as measured in the two spectra, and in a photometric exposure with the Boron filter on the Low Energy telescopes (through the ratio of measured X-ray flux to model flux at the stellar surface, using the distance, $63.3 \mathrm{pc}$, determined by Dahn et al. [1982]). Figure 3 shows these (99\%) constraints, as well as the constraints imposed by the visual magnitude estimate $V=12.99 \pm 0.03$, taken from Holberg et al. (1986). The intersection of the EXOSAT constraints and the 'optical' constraint gives the range of $T_{e}$ and $R / R_{\odot}$ that yields models consistent with both the X-ray and optical absolute flux from $\mathrm{HZ} 43$; we derive $T_{e}=45,000-54,000 \mathrm{~K}, R / R_{\odot}=0.0140-0.0165$. At $R / R_{\odot}=0.015$, the spectra measured with the EINSTEIN OGS and the Voyager 2 EUV spectrometer (Holberg et al. [1980]) yield effective temperatures that agree well with this solution (indicated at the top of Figure 3 ). Thus we determine the luminosity of $\mathrm{HZ} 43$ to be $L / L_{\odot}=1.0-1.5$. 

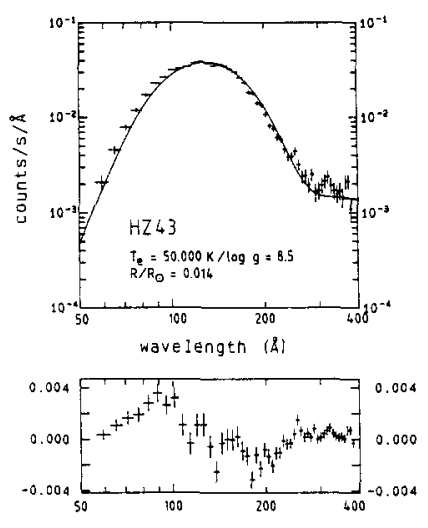

Figure 4. EXOSAT $5001 \mathrm{~mm}^{-1}$ TGS (3000LX) spectrum of $\mathrm{HZ} 43$, fitted with a model spectrum at $T_{e}=$ $50,000 \mathrm{~K}, R / R_{\odot}=0.014, \log g=8.5, \mathrm{He} / \mathrm{H}=0, n_{H}=2 \times$ $10^{18} \mathrm{~cm}^{-2}$, that is consistent with measured optical/UV fluxes with respect to total $\mathrm{X}$-ray flux. The spectral shape of the model is seen to exhibit a systematic difference with the measured shape of $15 \%$ in monochromatic flux shortward of $90 \AA$, indicative of a systematic uncertainty in the model spectra (see text).

When we fit model atmospheres spectra with parameters in the above ranges, however, we find that the shape of the model spectra does not match the shape of the measured spectrum at the shortest wavelengths $(\lambda<90 \AA) ; \chi^{2}$ indicates that the models do not yield a statistically acceptable description of the shape of the 3000LX spectrum. At $T_{e}=50,000 \mathrm{~K}$ we have to assume a $15 \%$ systematic error in the model fluxes shortward of $90 \AA$. This is shown in detail in Figure 4 (3000LX data, and a model at $T_{\mathrm{e}}=50,000 \mathrm{~K}, \log g=8.5, \mathrm{He} / \mathrm{H}=0, n_{H}=2 \times 10^{18} \mathrm{~cm}^{-2}, R / R_{\odot}=0.014$; reduced $\chi^{2}=5.70$; the lower panel shows the post-fit residuals). This systematic error cannot be traced to a systematic error in the calibration of the spectrometer, and must be ascribed to the model atmospheres calculations. This conclusion is confirmed by the fact that corresponding model atmospheres spectra, calculated with different codes based on the same input physics show $15 \%$ differences in monochromatic flux at the shortest wavelengths (these are the models published by Petre et al. [1986] and Wesemael et al. [1980], and our own calculations). Having verified the numerical accuracy of our own code, we tentatively identify a different treatment of the ionization balance as the origin of the observed differences in output of the three codes, the short-wavelength tail of the spectrum being extemely sensitive to the ionization balance.

At present the exact source of the differences remains unresolved, nor can any of the three codes be identified a priori as the one being free of the systematic uncertainties mentioned. However, the three codes agree in monochromatic flux longward of $100 \AA$, so that the $\mathrm{Al} / \mathrm{P}$ spectrum can be used to measure $\mathrm{He} / \mathrm{H}$ and $n_{H}$ independent of the specific code used to generate the models. In addition, the codes agree to within a few percent in integrated X-ray flux, so that our multicolor photometric determination of effective temperature and radius is valid, regardless of the systematic error diagnosed above. Improved accuracy in the estimate of $T_{e}$ for $\mathrm{HZ} 43$, and an estimate of $\log g$, using the shape of the measured X-ray spectrum has to wait until model atmospheres calculations appropriate to hot DA atmospheres are available, that are free of systematic uncertainties to the level of a few percent in monochromatic flux at the shortest wavelengths.

A full discussion of the HZ 43 data and their analysis can be found in Paerels et al. (1986b) and Heise et al. (1988).

references

Dahn, C. C., et al. (1982), Astron. J. , 87,419 .

de Korte, P. A. J., et al. (1981), Space Sci. Rev. , $30,495$.

Heise, J., et al. (1988), Ap.J. , ( in press).

Holberg, J. B., et al. (1980), Ap.J.(Letters), 242 , L119.

Holberg, J. B., Wesemael, F., and Basile, J. (1986), Ap.J. , 306 , 629.
Paerels, F. B. S., et al. (1986a), Ap.J.(Letters), 309 , L33.

Paerels, F. B. S., et al. (1986b), Ap.J., $308,190$.

Paerels, F. B. S., et al . (1988), Ap.J., $329,849$.

Petre, R., Shipman, H. L., and Canizares, C. (1986), Ap.J., $304,356$.

Taylor, B. (1985), Adv. Space Res. , 5 , 35.

Wesemael, F., et al. (1980), Ap.J.Suppl. , $43,159$. 